\title{
Is Temporary Proximal Artery Clipping in Arteriovenous Malformation Surgery Safe?
}

\author{
Balaji PAI ${ }^{1}$, Nagarjun Maulyavantham NAGARAJ', Sathyaranjandas HEGDE² \\ ${ }^{1}$ Bangalore Medical College and Research Institute, Department of Neurosurgery, Bangalore, India \\ ${ }^{2}$ M.S. Ramaiah Medical College, Department of Neurosurgery, Bangalore, India
}

This study has been presented as an oral presentation at the XVI World Congress of Neurosurgery (WFNS) between 20 and 25 August, 2017 at Istanbul, Turkey.

Corresponding author: Nagarjun Maulyavantham NAGARAJ nagarjunmn@gmail.com

\section{ABSTRACT}

AIM: To describe the authors' experiences with surgical excision of cerebral arteriovenous malformations (AVMs) using temporary proximal artery clipping to reduce intraoperative bleeding and excision time.

MATERIAL and METHODS: Temporary proximal artery clipping was employed by the authors during surgery on 14 patients with cerebral AVMs in a public-sector institution. Seven AVMs were situated in eloquent areas like the sensory, motor, and speech areas and the other seven were located in non-eloquent areas. Four patients each were classified as Spetzler grades 2 and 4 while three patients each were classified as grades 3 and 5. A large craniotomy flap was fashioned to enable access to the proximal feeding arteries along with the AVM. After wide dural opening, the proximal feeding arteries were first approached. The proximal middle cerebral artery was approached by opening the proximal sylvian cistern, an interhemispheric approach was employed for the distal anterior cerebral artery, and a subtemporal route was used for the P2 portion of the posterior cerebral artery. After placing a temporary aneurysm clip on the proximal feeding artery, excision of the AVM was performed, employing the basic principles of AVM excision.

RESULTS: Total excision of the AVM was achieved in all cases. There were no instances of cerebral infarction or neurological deficits attributable to temporary clipping of the proximal artery. There was no mortality in this series.

CONCLUSION: Temporary proximal artery clipping helps in surgical excision by reducing intraoperative bleeding and resection time.

KEYWORDS: Arteriovenous malformation, Brain, Excision, Temporary artery clipping

\section{INTRODUCTION}

$\mathrm{S}$ urgical excision of arteriovenous malformations (AVMs) in the brain was associated with enormous pessimism in the early part of the twentieth century. Although challenging, most of the pathologies can be treated safely and effectively with microneurosurgery (1). Surgical excision of AVMs has been rendered safer by various technological advancements like operating microscopes, better imaging techniques, microinstruments, preoperative embolization, intraoperative monitoring, and postoperative management (3). Despite these advancements, surgery for the lesions still poses a challenge, with uncontrolled bleeding and brain swelling being dreaded intraoperative complications.

The intent of this article is not to review and suggest management strategies for cerebral AVMs. The authors describe their experiences with surgical excision of cerebral AVMs using temporary proximal artery clipping. This simple procedure significantly reduced intraoperative bleeding, brain swelling, and operative time during surgery in this series. The objective of this article is to focus on this innovative and simple procedure

Balaji PAI (1) : 0000-0001-6021-069X Nagarjun Maulyavantham NAGARAJ (1) : 0000-0003-1526-0238 Sathyaranjandas HEGDE (10) :0000-0003-3315-9563 
and assess its safety both clinically and radiologically. To the best of our knowledge, there is no mention of the technique in literature.

\section{MATERIAL and METHODS}

This is a single center prospective study undertaken by the authors in a public-sector tertiary care teaching institution. Temporary proximal artery clipping was performed by the first author during surgery on 14 patients with cerebral AVMs between February 2015 and August 2017. Nine patients were male and five were female. The ages of the patients ranged from 7 to 46 years, as shown in Table I. Three patients had a history of previous hemorrhage, five presented with refractory seizures, four with progressive neurological deficits, one with altered sensorium, and the remaining four with severe headache. The neurological deficits could be attributed to the cerebral steal phenomenon as well as the location and mass effect of the AVMs. Seven AVMs were situated in eloquent areas like the motor, sensory, and speech areas while seven were located in non-eloquent areas. The size of the nidus was generally large in our series, with a size larger than $6 \mathrm{~cm}$ in three patients, between 3 and $6 \mathrm{~cm}$ in 10 patients, and less than 3 $\mathrm{cm}$ in only one patient. Nine AVMs had two arterial feeders and five had a single arterial feeder. None of the patients in our series had more than two arterial feeders (Figure 1). The compactness of the nidus was also evaluated using Digital Subtraction Angiography (DSA) and subsequently confirmed intraoperatively. Eight patients had a compact nidus, a diffuse nidus was noted in five patients, and one patient had a partly compact and partly diffuse nidus. Only superficial draining veins were present in eight patients while additional deep venous drainage was noted in six patients. Four patients were classified as Spetzler grade 2, three as grade 3, four as grade 4 , and three as grade 5.

All the patients underwent DSA in addition to computed tomography $(\mathrm{CT})$ /magnetic resonance (MR) angiography after preliminary $C T$ and magnetic resonance imaging (MRI) scans (Figures 2; 3A, B; 4A, B; 5A, B). Functional MRI was performed in two patients. No feeding artery flow-related aneurysms were noted in the DSAs of these patients. Blister feeding artery aneurysms were observed intraoperatively in three patients but no attempts were made to treat them. Intranidal aneurysms were observed in four patients and a large venous varix was seen in one patient.

The indications for surgery in this series consisted of progressive neurological deficit in four patients, refractory seizures in

Table I: Master chart depicting all the patients with the various proximal arteries clipped, their respective clipping times and follow-up modified Rankin's scale scores (score 0 is no symptoms and score 6 is dead).

\begin{tabular}{|c|c|c|c|c|c|c|}
\hline $\begin{array}{c}\text { Case } \\
\text { no }\end{array}$ & $\begin{array}{l}\text { Age (years), } \\
\text { Sex }\end{array}$ & Site of AVM & $\begin{array}{l}\text { Spetzler } \\
\text { Grade }\end{array}$ & Clipping Site & $\begin{array}{l}\text { Clipping Time } \\
\text { (Minutes) }\end{array}$ & $\begin{array}{l}\text { Modified Rankin's } \\
\text { Scale score at } \\
\text { 6- month follow-up } \\
\text { time point }\end{array}$ \\
\hline 1 & $40, \mathrm{M}$ & Right Temporal & 2 & $\begin{array}{c}\text { Right MCA Inferior division, } \\
\text { Right P2 }\end{array}$ & MCA: 31 PCA: 50 & 0 \\
\hline 2 & $23, \mathrm{M}$ & Left Sylvian & 5 & Left MCA, Superior division & 59 & 0 \\
\hline 3 & $44, \mathrm{M}$ & Right Temporal & 4 & Right MCA, Superior division & 80 & 0 \\
\hline 4 & $25 / F$ & Right Parietal & 3 & Right MCA, Superior division & 58 & 3 \\
\hline 5 & 39, M & Right Temporal & 2 & Right P2 & 20 & 0 \\
\hline 6 & 29, F & Right Parietal & 4 & Right Cortical MCA, ACA & 57 & 0 \\
\hline 7 & $46, F$ & Right Temporooccipital & 4 & Right P2 division & 70 & 2 \\
\hline 8 & 42, M & Right Peri-Rolandic & 5 & Right M1, A3 & MCA: 48 ACA: 38 & 3 \\
\hline 9 & $12, \mathrm{M}$ & Left Frontoparietal & 2 & M1 & 45 & 0 \\
\hline 10 & $7, \mathrm{M}$ & Left Occipital & 3 & P2 & 45 & 0 \\
\hline 11 & $28, F$ & Right Frontoparietal & 5 & $\mathrm{M} 1, \mathrm{~A} 2$ & $\begin{array}{l}\text { M1:133 } \\
\text { A2:120 }\end{array}$ & 3 \\
\hline 12 & $16, \mathrm{M}$ & Right Frontal & 2 & $\mathrm{~A} 2$ & 48 & 0 \\
\hline 13 & $32, \mathrm{~F}$ & $\begin{array}{l}\text { Left Frontal } \\
\text { Broca's Area }\end{array}$ & 3 & M1 & 52 & 2 \\
\hline 14 & $28, \mathrm{M}$ & Right Sensory Cortex & 4 & M5, A4 & $\begin{array}{l}\text { A4: } 40 \\
\text { M5: } 55\end{array}$ & 3 (at 3 months) \\
\hline
\end{tabular}

ACA: Anterior cerebral artery, F: female, M: male, MCA: middle cerebral artery. 
Pai B. et al: Temporary Proximal Artery Clipping in AVM

five patients, risk of rupture in three patients, persistent altered sensorium in one patient, and severe headache in one patient. Detailed informed consent regarding the procedure and pos-

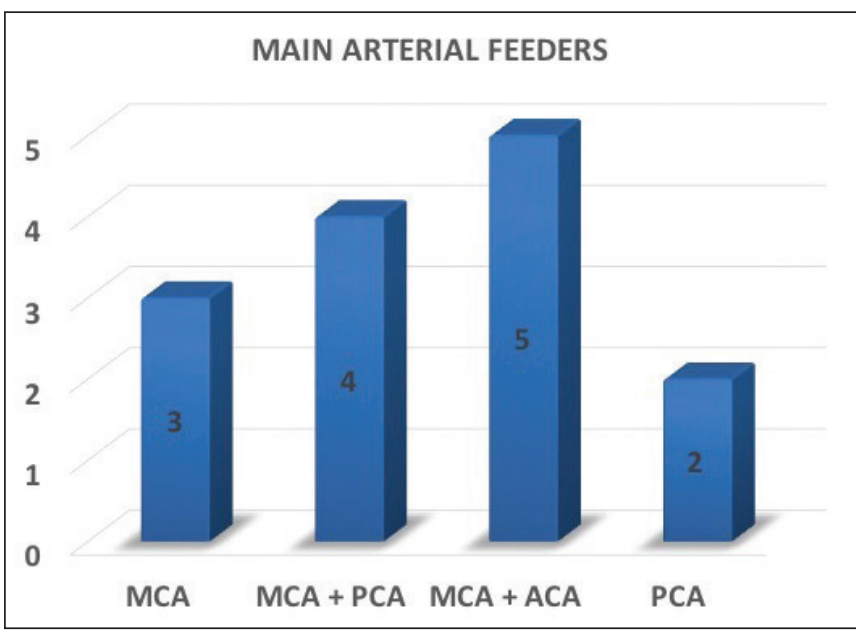

Figure 1: The number and types of main arterial feeders to the AVM noted in this series (ACA: anterior cerebral artery, MCA: middle cerebral artery, PCA: posterior cerebral artery). sible complications was obtained from all patients and their close relatives prior to surgery. Ethical committee approval was not required as standard operative procedures were followed in this study.

\section{Procedure}

The patients were induced after deep anesthesia and were positioned in consideration of the site of the AVM. Patients were positioned in such a manner that the proximal arteries could be approached along with the AVM. Patients with temporal AVMs were placed supine with the head turned to the opposite side whereas occipital AVMs were approached with the patient in the prone position. A large craniotomy flap was fashioned so that the proximal arteries could be reached along with the AVM. After a wide dural opening, the proximal arteries were first approached. The proximal middle cerebral artery (MCA) was approached by opening the proximal sylvian cistern (Figure 6), the distal anterior cerebral artery (ACA) was approached interhemispherically (Figure 7), and the P2 portion of the posterior cerebral artery (PCA) was approached through a subtemporal route. The M1 portion of the MCA was clipped close to its terminal end to avoid incorporation of the lenticulostriate arteries. The P2 portion of the PCA was usually clipped in the crural cistern where it is easily visualized. After
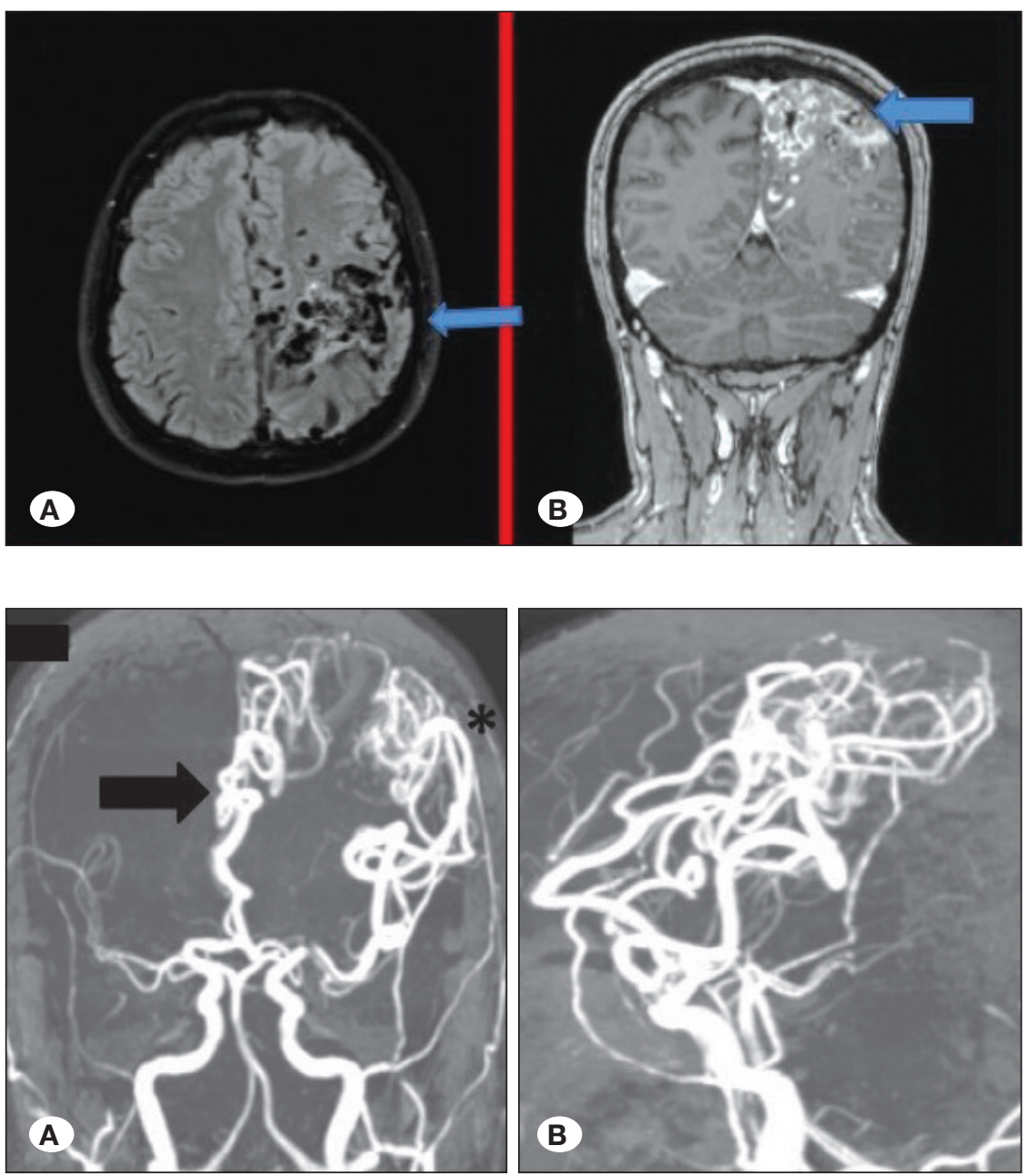

Figure 2: MRI scan (A: FLAIR axial image and B: gadolinium enhanced coronal image), of a patient showing a large AVM in the eloquent (left motor) cortex (indicated with arrows).

Figure 3: MR angiogram of the patient in Figure 2 showing the AVM fed by the left ACA (arrow) and MCA (asterisk) in AP (A) and lateral views (B) (ACA: anterior cerebral artery, MCA: middle cerebral artery). 
Pai B. et al: Temporary Proximal Artery Clipping in AVM
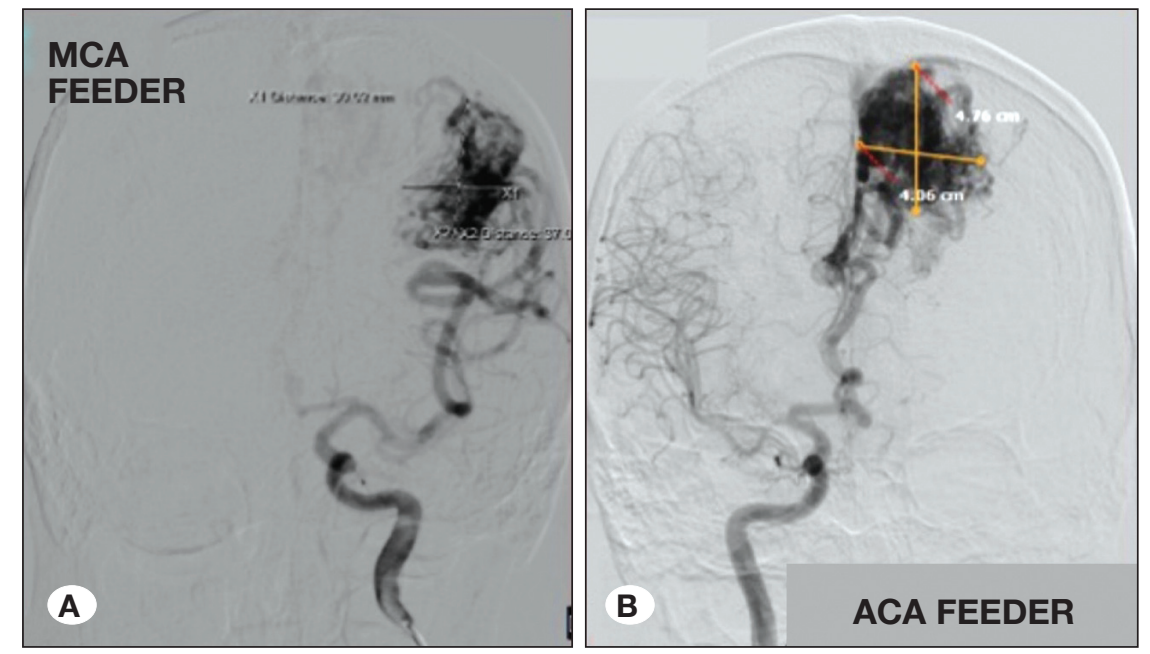

Figure 4: Digital subtraction angiography of the patient in Figure 2 showing the AVM in the arterial phase with the lateral part fed by the middle cerebral artery (A) and the medial part fed by the anterior cerebral artery (B).
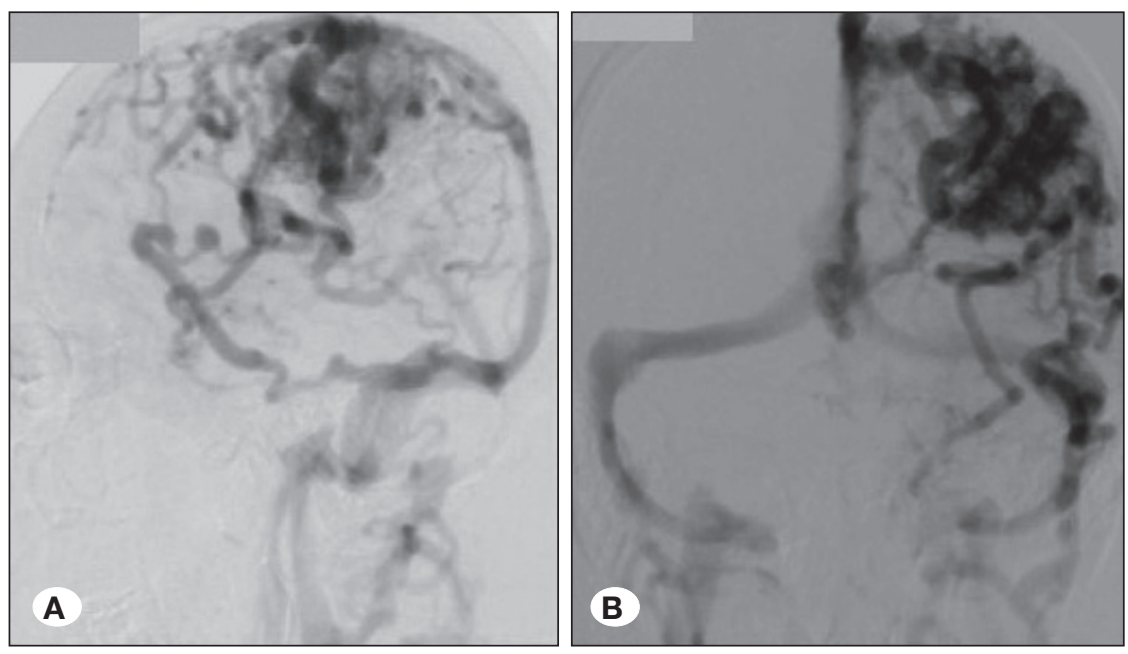

Figure 5: Digital subtraction angiography of the patient in Figure 2 showing the AVM in the venous phase with veins draining into both the superficial (superior sagittal sinus) and deep venous system (straight sinus) in lateral (A) and AP views (B).

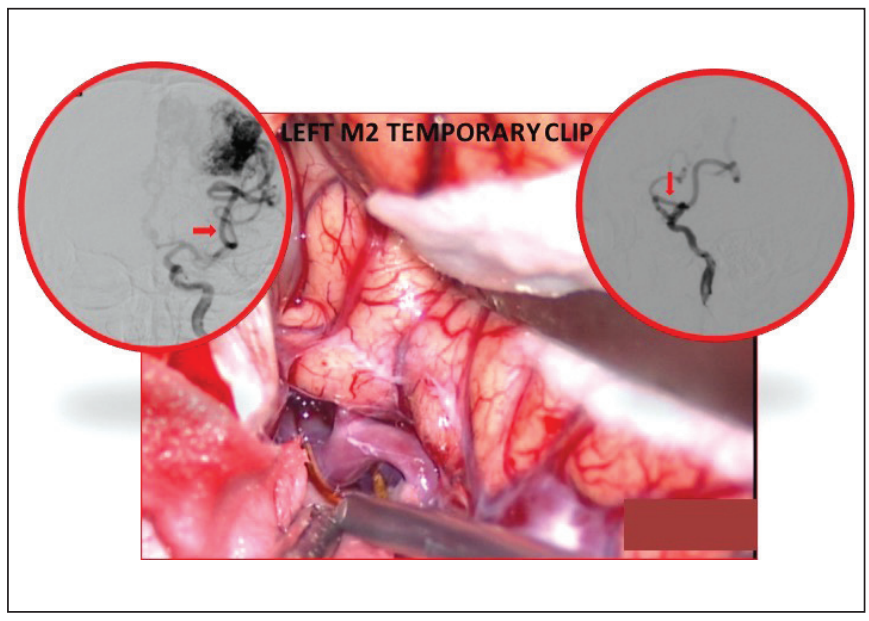

Figure 6: A temporary clip being placed on the superior trunk of the left MCA (M2) of the patient in Figure 2 (MCA: middle cerebral artery).

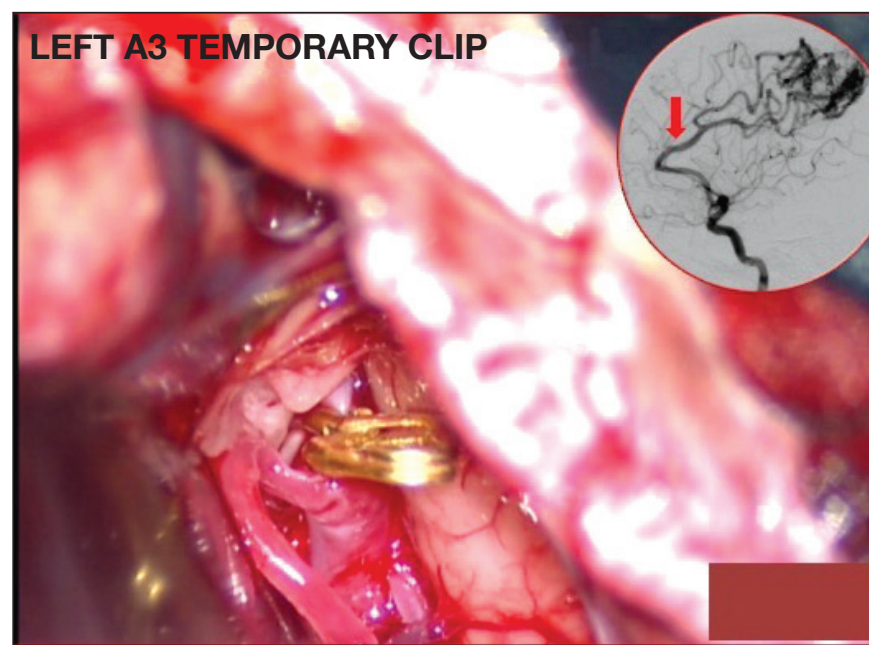

Figure 7: A temporary clip placed on the left ACA (A3) of the patient in Figure 2 (ACA: anterior cerebral artery). 
placing a temporary aneurysm clip on the proximal artery, excision of the AVM was performed. In one patient, the ACA was temporarily clipped at A4 and the MCA at M5 (distal temporary clipping close to the nidus). While the temporary clips were in place, standard brain protection techniques were employed such as mannitol, steroids, and propofol infusion. Normotension was preferred during this period and hypotension was avoided. A cortical incision was made close to the AVM nidus and the gliotic plane around the nidus was entered. Most AVMs could be identified on the cortical surface. The AVM nidus was excised completely in all cases following the general microsurgical principles for AVM excision. Hemostasis was achieved and the temporary clips were removed. The blood pressure was elevated and hemostasis confirmed. We had no difficulty achieving hemostasis in our series and closure was completed in the usual manner. No attempt was made to close the dura in a watertight fashion. The senior author, who is an advocate of intraoperative angiography in aneurysmal surgery especially for giant aneurysms, did not find it necessary in this series. However, intraoperative angiography may be useful for identifying any residual "hidden" nidus. The temporary clipping time varied from 20 to 133 minutes (Table I). The temporary clip was not released during excision of the nidus in any of the patients.

Postoperative management was directed toward hypotension induced by propofol infusion. With the exception of four grade 2 patients, the other twelve patients were electively ventilated along with hypotension for 72 hours. Subsequently, a quick and smooth extubation was done to avoid hypertensive peaks. Postoperative CT was performed the next morning in all cases (generally after 18 hours of surgery). Hounsfield values between 15 and $20 \mathrm{HU}$ in a particular arterial territory were considered to be indicative of an infarct. Three patients who experienced an increase in neurological deficits also underwent diffusion weighted MRI to rule out an infarct. All patients underwent a CT cerebral angiogram after six weeks (Figures 8) and the follow up period ranged from three months to 24 months.

\section{RESULTS}

Total excision of the AVM was achieved in all cases as confirmed by postoperative CT cerebral angiogram. There were no instances of cerebral infarction attributable to the temporary clipping in our series as evidenced by postoperative CT scan in all cases and by diffusion weighted imaging (DWI) $\mathrm{MRI}$ in selected cases. There was no case of normal perfusion pressure breakthrough in our series. One patient developed venous infarction of the temporal lobe due to inadvertent compromise of the Vein of Labbé. This patient recovered from surgery but showed neurological deterioration after 48 hours. A decompressive craniectomy was performed, followed by uneventful recovery and subsequent bone flap replacement after three months. Three patients with motor area AVMs developed postoperative hemiplegia, which recovered to minimal hemiparesis over six months.

One of these patients also developed immediate postoperative aphasia, which recovered to normalcy within three months. Surprisingly, the only patient with Broca's area AVM had minimal postoperative speech deficits, which also recovered completely within six weeks. Postoperative deficits in these three patients were due to the excision of the nidus from an eloquent area (motor cortex) and not due to the effects of temporary proximal artery clipping as demonstrated by the postoperative DWI MRI. One patient who presented with altered sensorium improved and is independent with minimal hemiparesis. A modified Rankin's scale was used to assess the outcome at six months (Table I). There was no mortality in our series.

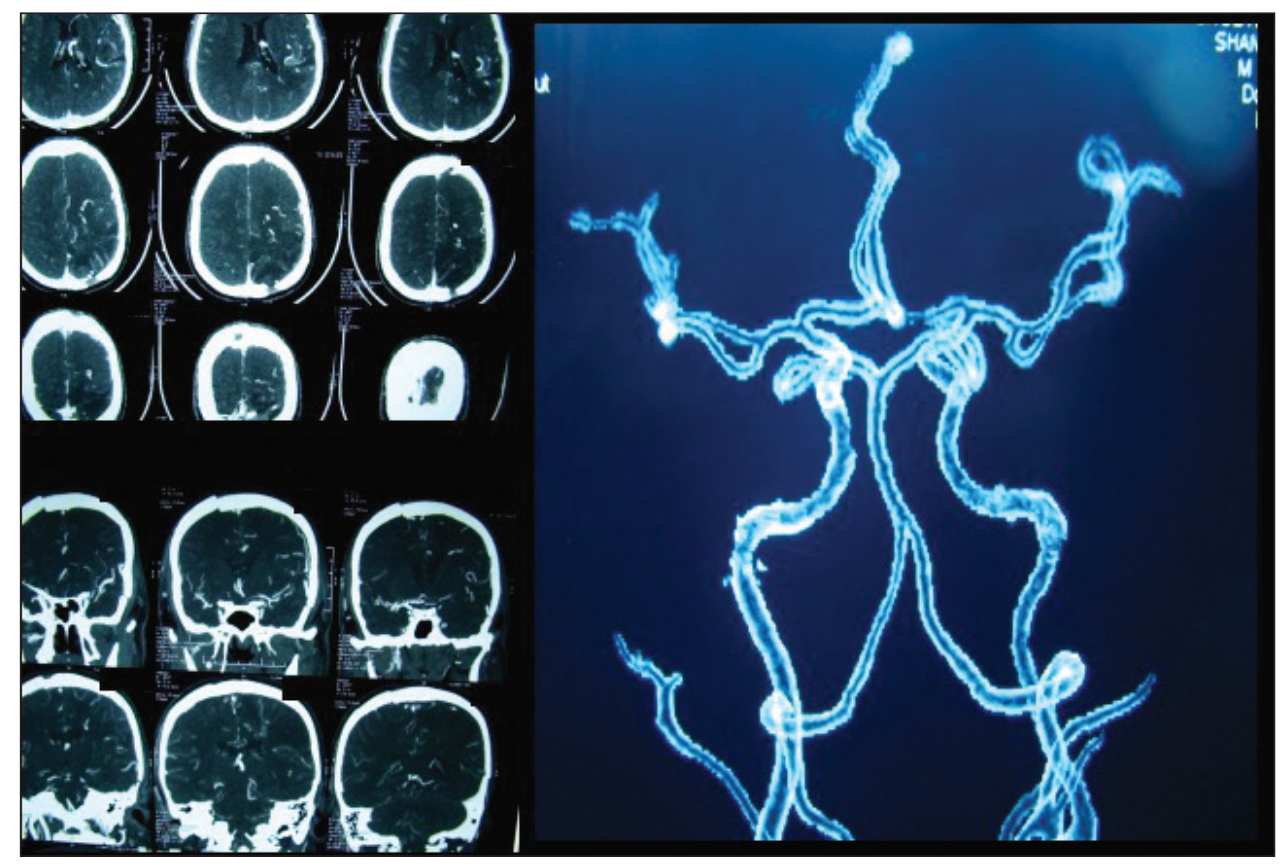

Figure 8: Postoperative CT angiogram of the patient in Figure 2 with a large left motor cortex depicting total excision. 


\section{DISCUSSION}

Success in the excision of AVMs requires a firm commitment toward formulating and adhering to a definite operative plan after viewing the entire neuroimaging profile including DSA, $\mathrm{CT} / \mathrm{MR}$ angiogram, functional MRI, MR tractography, etc. General strategies for AVM excision include helical dissection of the nidus, coagulating/clipping and cutting all the arterial feeders close to the nidus and subsequent obliteration of venous drainage.

Hashimoto et al advocated proximal feeding artery clipping with no adverse ischemic effects (3). However, their description suggests that the feeding arteries were clipped at a more distal location than in our series, in which we clipped M1, M2, P2, A2, A3, and A4. Similar distal feeding artery clipping has also been described by other surgeons $(1,4,6)$. Kandel reported clipping the main arterial feeder of an AVM through a burr hole in select cases prior to radical excision at the beginning of surgery (5). In our series, proximal arteries were clipped in the sylvian cistern (M1 and M2), crural cistern (P2), and interhemispheric cistern (A2, A3, and A4). Temporary distal feeding artery clipping close to the nidus is always preferable to proximal artery clipping. The authors also prefer to clip the feeding artery as close to the nidus as possible if the arteries can be easily visualized intraoperatively. However, visualization of feeding arteries close to the nidus may be difficult as they are usually subcortical. Navigation may be required to identify these vessels. Inadvertent entry or injury to the nidus while dissecting these vessels will lead to troublesome bleeding. Proximal artery temporary clipping avoids all these difficulties.

\section{Benefits of Temporary Proximal Artery Clipping}

The benefits of temporary proximal artery clipping are multifaceted and comprehensive. The direct effect of performing this simple procedure early in the surgery is apparent during the dissection of the nidus. Intraoperative bleeding is significantly reduced and the nidus is also noted to be softer and less vascular. Draining veins also become darker, inducing a sense of confidence in the surgeon. The excision time for the AVM also decreased drastically after the authors adopted this procedure. The excision time, which is identical to the temporary clipping time, varied from 20 to 133 minutes. Moreover, no intraoperative brain swelling was noted due to this procedure. The disadvantages include substantially larger craniotomy and intraoperative minor position changes to access the arteries as well as the nidus.

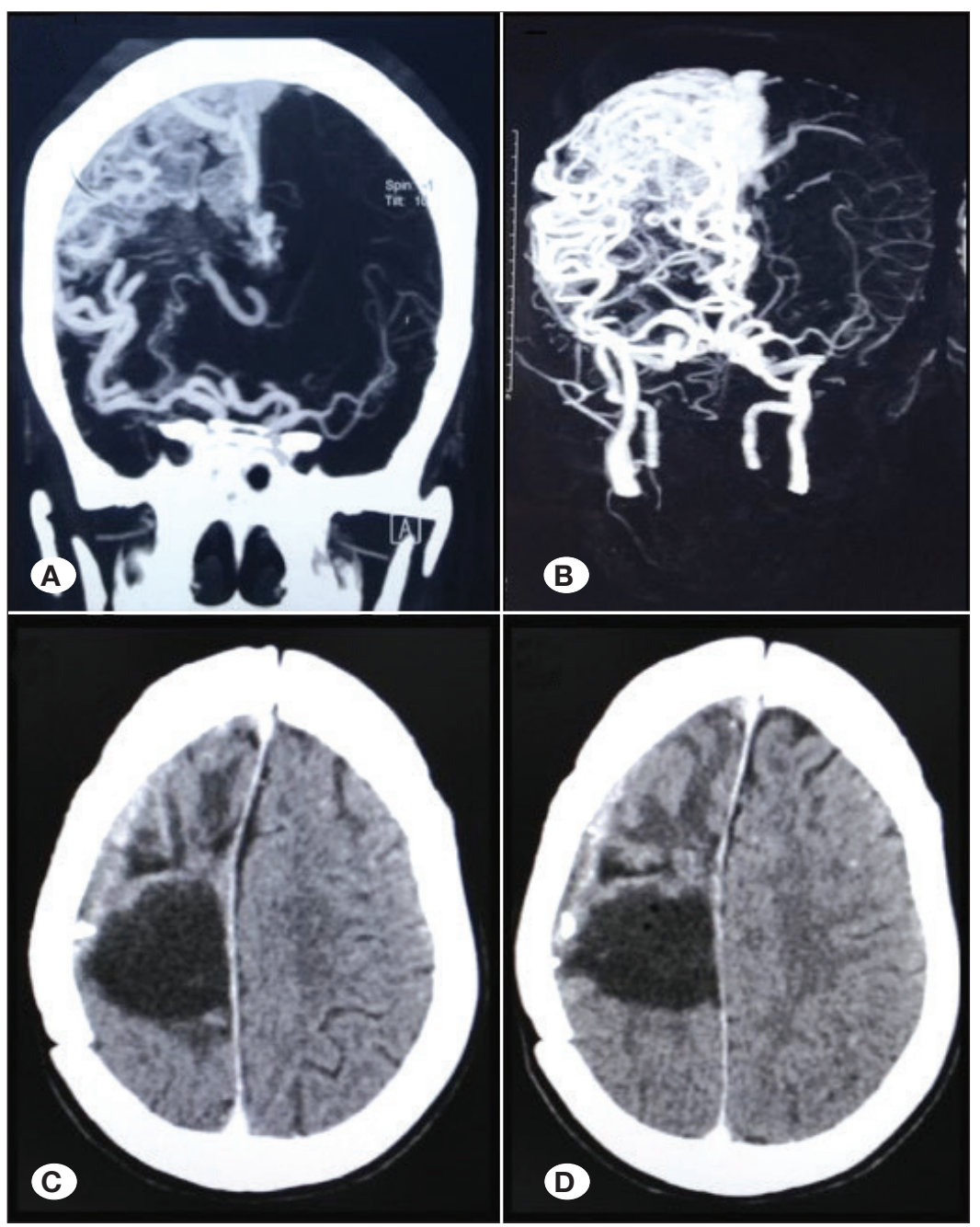

Figure 9: CT angiogram (A, B) of another patient with a large AVM in the right motor cortex with a diffuse nidus and fed by the right MCA and ACA. Postoperative CT scan (C, D) shows total excision of the AVM (ACA: Anterior Cerebral Artery, MCA: Middle Cerebral Artery). 


\section{Pathophysiology of AVM that Facilitates Temporary Proximal Artery Clipping}

AVMs are congenital vascular malformations but also change dynamically in their pathologies during their lifetime (3). Cerebral blood flow measurements in the brain surrounding the AVM suggest a certain degree of hypoxia and ischemia, which is chronic and long standing $(2,4)$. Proximal clipping of the feeding artery may result in low perfusion distal to the clip. However, our results showed no cerebral infarction attributable to temporary clipping. This may be considered a paradox and seems to suggest that circulatory adaptive mechanisms are already in effect in these areas due to the "steal" phenomenon. Hence, prolonged temporary clipping periods were well tolerated by the brains of our patients. This has also been the experience of other surgeons $(3,4)$. Another factor that may have contributed to this phenomenon is the alacrity with which the nidus was excised, leading to increased blood flow to the adjacent brain. This cannot be compared to temporary clipping in aneurysmal surgery where such prolonged periods will not be tolerated. Adjacent brain oxygenation also increases as the AVM excision progresses (2). This was our experience as well, albeit by visual perception, wherein the surrounding brain would turn deep pink and even red as excision of the AVM nidus was carried out. The authors wish to suggest that a chronically ischemic brain can tolerate diminished cerebral blood flow far better than a normal brain due to the adaptive mechanisms of the microvasculature and the brain. This is analogous to the phenomenon of recruitment practiced during temporary parent arterial clipping for aneurysm surgery. Our study would have been more valuable if intraoperative cerebral blood flow or tissue oxygenation measurements had been carried out. Unfortunately, lack of appropriate logistics in our institute prevented these measurements. The authors consider this a possible limitation of the study. Sano et al.(6) was also in agreement, having had a similar experience with temporary proximal artery clipping in their earlier surgical periods. Intraoperative angiography may be useful, especially for revealing concealed portions of the nidus that may be missed during resection. However, this technique was not employed in our series although we had prepared for it in three cases. The intraoperative impression in these cases was sufficiently convincing to reject intraoperative angiography.
Three patients with a large AVM involving the motor cortex experienced immediate postoperative neurological deficits but recovered significantly within six months. This increase in neurological deficits can be attributed to the resection of the nidus from the motor cortex and was not due to cerebral infarction (Figure 9). Surprisingly, even a Broca's area AVM (confirmed by functional MRI) showed minimal postoperative deficits. This reveals the forgiving nature of the brain in an AVM due to chronic ischemia.

\section{CONCLUSION}

Temporary proximal artery clipping is a simple, safe yet effective method to reduce intraoperative blood loss and brain swelling as well as expedite AVM excision surgery. No incidence of cerebral infarction attributable to temporary clipping was noted in this series. This innovative step instills confidence in the surgeon during AVM excision including high grade AVMs.

\section{REFERENCES}

1. Bhatoe HS: Operative nuances of surgery for cortical arteriovenous malformations: A safe solution and permanent cure. Neurol India 64 Suppl:S101-109, 2016

2. Charbel FT, Hoffman WE, Misra M, Ausman JI: Increased brain tissue oxygenation during arteriovenous malformation resection. Neurol Med Chir 38 Suppl:171-176, 1998

3. Hashimoto N, Nozaki K, Takagi Y, Kikuta K, Mikuni N: Surgery of cerebral arteriovenous malformations. Neurosurgery 61:375-387; discussion 387-379, 2007

4. Hernesniemi J, Romani R, Lehecka M, Isarakul P, Dashti R, Celik O, Navratil O, Niemela M, Laakso A: Present state of microneurosurgery of cerebral arteriovenous malformations. Acta Neurochir Suppl 107:71-76, 2010

5. Kandel El: Clipping of an AVM arterial feeder through a burr hole prior to radical surgery. Technical note. J Neurosurg 66:305-306, 1987

6. Sano H, Kato Y, Bannur U, Okuma I, Kanaoka N, Kanno T: Strategy for the treatment of arteriovenous malformations. $J$ Clin Neurosci 7 Suppl 1:60-68, 2000 